First publ. in: Berichte der Bunsen-Gesellschaft 98 (1994), 3, pp. 443-446

\title{
Transport Phenomena in Physisorbed Films: Investigations with Optically Excited Surface Plasmons
}

\author{
S. Herminghaus, U. Sigel, T. Paatzsch, H. Musil, and P. Leiderer \\ Fakultät für Physik, Universität Konstanz, D-78434 Konstanz, Germany
}

\author{
Key Words: Diffusion / Interfaces / Liquids / Transport Properties / Viscosity
}

\begin{abstract}
Experiments were performed on the latcrai transport kinetics of wetting layers. Oprically excited surface plasmons were used to study both the spreading behaviour of silicon oil and the equilibration dynamics of ethanol. The precursor film profiles of silicon oil were found to agree well with the theory of viscous flow of nonvolatile liquids. For ethanol, evaporation effects and viscous transport could be observed separately. Apparently, the viscous transport is suppressed by almost two orders of magnitude. Possible explanations are discussed.
\end{abstract}

A lot of work has been dedicated to the transport kinetics of viscous, nonvolatile liquids on surfaces $[t-5]$, i.e., the spreading of liquid drops. For systems in which evaporation plays a significant role, experiments become more difficult, since it may be necessary to separate different transport mechanisms in the analysis. Consequently, systematic measurements of lateral transport in wetting layers of volatile liquids have not been performed due to the lack of suitable experimental techniques. In the present work, the transport dynamics of thin films of both poly-dimethyl-siloxane (PDMS), a viscous fluid which is only slightly volatile $[3,8]$, and ethanol is studied using optically excited surface plasmons. In this technique, one exploits the fact that the phase velocity of the surface plasmons propagating on the metallic substrate depends very sensitively on the thickness of the adsorbed layer. By attenuated total reflectance, surface plasmons can be resonantly excited by a laser beam at a certain angle of incidence corresponding to the phase velocity of the surface plasmons and thus to the adsorbed layer thickness $[6,7]$.

For the studies of the spreading behaviour of PDMS, we performed so-called vertical rise experiments. At time $l=0$, the vertical substrate was put in a trough containing some PDMS [8], which wets metal surfaces completely $[1,3]$. We then observed the wetting layer of PDMS creeping up the

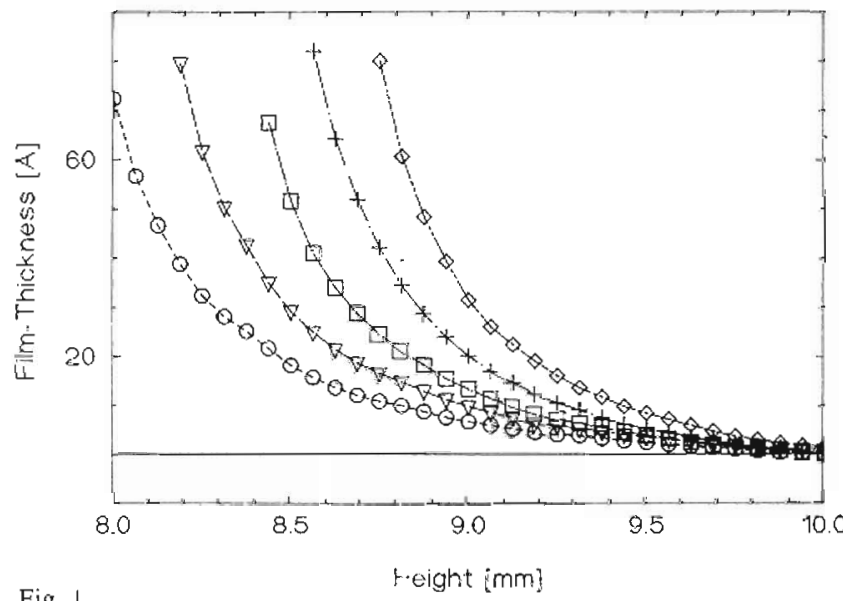

Precursor film profiles of upward creeping PDMS film for five different times $(10 \mathrm{~h}, 10.5 \mathrm{~h}, 11 \mathrm{~h}, 11.5 \mathrm{~h}, 12 \mathrm{~h})$ after lirst contact of the sample with the PDMS reservoir substrate by vertically scanning a focused laser beam over the sample and measuring the angular position of the surface plasmon resonance as a function of height. The beam was focused down to a width of $30 \mu \mathrm{m}$ which determines the spatial resolution of the experiment. The thickness resolution was $\approx 0.5 \AA$.

Figure 1 shows a set of scans demonstrating the upward creep of the uppermost tip of the film, the so-called precursor film, on a gold surface. The times at which the scans were taken are $10.0,10.5,11.0,11.5$ and $12.0 \mathrm{~h}$ after contact with the PDMS bath. Obviously, the profile of this part. of the film is almost independent of time. The theory of viscous spreading of nonvolatile liquids predicts that for Poiseuille flow with no-slip condition, the film thickness $d$ should scale as $d \propto x^{-2}$ in the investigated thickness range, where $x$ is the distance measured along the substrate [9]. To compare our data with this prediction, we plotted the profiles on a log-log scale in Fig. 2 . The straight lines fitted to the data have a slope of $1.98 \pm 0.02$, in perfect agreement with the abovementioned theory (the error represents the confidence level of the fit). This is remarkable for two reasons. First, the substrate was an evaporated gold film

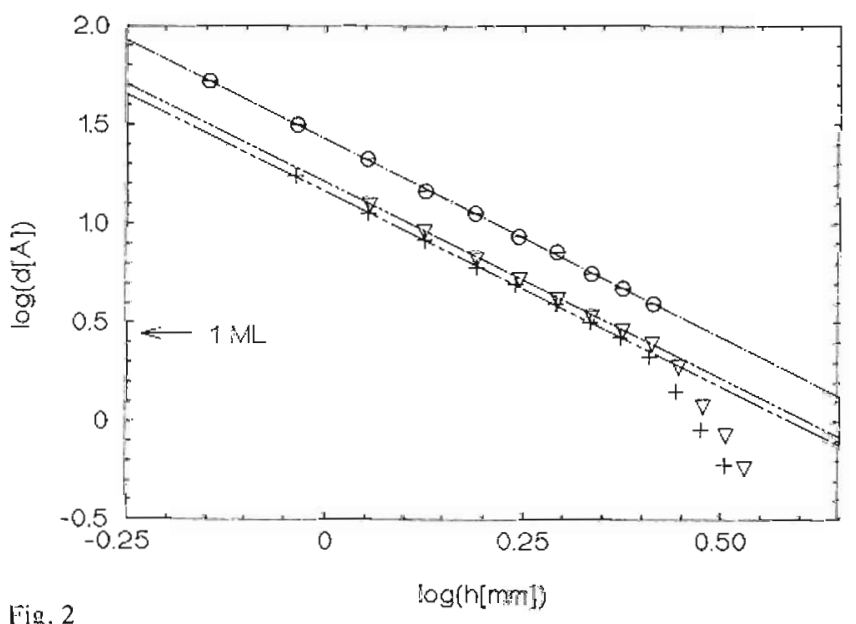

Log-log plot of film thickness vs. height for three representative profiles. The slope of the straight lines is represented by $1.98 \pm 0.02$, in good agreement with theory. The transition between mesoscopic and molecular regime can be scen. It occurs at a thickness of $\approx 7 \mathrm{~A}$ corresponding to a flat, compact monolayer 
with a rms roughness of some tens of $\AA$, as revealed by scanning tunneling microscopy [10]. Thus, the roughness amplitude is in the same order of magnitude as the film thickness. In spite of this fact, the "scaling exponent" of the profile is just as predicted for a flat surface. Secondly, it has been reported previously that even the slight volatility of this kind of silicon oil can lead to Marangoni effects in the contact region, which strongly affect the spreading dynamics, leading to accumulation of fluid in the contact region. This does not seem to be the case in our experiment.

The apparent absence of Marangoni effects can be roughly understood, however, since these have been observed only at larger thicknesses, where van-der-Waals interactions are negligible. The critical thickness below which Marangoni effects may become important scales as the inverse square root of the gravitational force [11]. In our case, the latter is to be replaced by the van-der-Waals force at thicknesses below $100 \AA$, which is about nine orders of magnitude stronger than gravity. Thus, since the reported critical thickness for our material is $14 \mu \mathrm{m}$ in the gravitational regime, the expected critical thickness under vander-Waals force is $4.4 \AA$. This is less than the backbone diameter of the PDMS molecules, which is about $7 \AA$. In this regime, a strong deviation from the theoretical prediction (towards lower coverage) is indeed observed, in accordance with earlier findings on sub-monolayer diffusion of PDMS in which the shapes of microscopic spreading droplets were studied [3]. However, since here we are dealing with individual molecules instead of continuous material, Marangoni effects are not the appropriate paradigma for the interpretation. Furthermore, it has been shown previously that there is virtually no 3D-evaporation of the first PDMS monolayer, and the experimental results could be very well modelled by Monte-Carlo simulation of a nonvolatile sub-monolayer [3].

The results point towards a coverage-dependent diffusivity which decreases when the coverage is decreased. This can be qualitatively understood from the two-dimensional excluded-volume interaction of the polymer molecules, which are known to lie flat on the surface [3]. The proximity of neighboring molecules gives rise to a reduction in configurational entropy and thus to an increase in the chemical potential. Hence a gradient in coverage invokes an additional gradient in the chemical potential, adding to the driving force for the collective diffusion. The closer the twodimensional packing, the larger is this effect, hence the diffusivity increases when the coverage is increased.

If one now considers wetting layers of volatile liquids, one is faced with a more complicated transport behaviour, since direct exchange of molecules with the vapour may play an important role. We exploited the fact that a small, spatially periodic thickness modulation of the film should decay exponentially with a decay time constant $A \propto q^{r}$, where $q$ is the wavenumber of the film thickness modulation and $v$ is characteristic of the dominant transport mechanism. For instance, direct exchange of molecules with the vapour phase leads to $v=0$, lateral diffusion within the film to $v=2$. Variation of $q$ and observation of the scaling behaviour of $A(q)$ allows to identify the dominant mechanism.

The spatial thickness modulation of the film was created by thermal desorption using two crossed beams from a Qswitched Nd: YAG laser. From the thermal properties of the sample (silver film evaporated onto glass) one can estimate that the amplitude of the spatial temperature profile is less than $10 \mathrm{mK}$ after $40 \mu \mathrm{s}$, and the overall heating of the surface is less than $1 \mathrm{~K}$ above equilibrium temperature after $1 \mathrm{~ms}$. Typical modulation amplitudes are in the range of a few monolayers. Experiments were performed with wetting layers of ethanol in the temperature range from $145-200 \mathrm{~K}$. The absolute value of the film thickness could be measured by means of the surface plasmon resonance position to be $\approx 30 \AA$ under the conditions of our experiment.

Due to the low binding energy of the wetting layers and the presence of different competing transport mechanisms, typical decay times are expected to be much shorter than the time needed to perform a scan over the surface. Thus, we spatially modulated the intensity of the surface plasmons by interference [12], with a period identically to that of the film thickness modulation. Depending on the relative phase of the surface plasmon intensity pattern and the thickness modulation, the film thickness is measured either in the "valleys" or on the "hills" of the modulation. The difference of both thickness values yields the modulation amplitude, which is monitored as a function of time.

For different temperatures we measured the decay constant $A$ as a function of the modulational wave number, $q$. Results for $T=170 \mathrm{~K}$ are plotted in Fig. 3. The scattering of the data points (on the order of $10-20 \%$ ) is probably due to small drifts in the film thickness. We see clear indication for a behavior of the form $A=a q^{2}+b$, which is expected if both transport mechanisms (lateral diffusion and exchange with the vapour phase) are present. For the coefficient $b$, kinetic gas theory yields

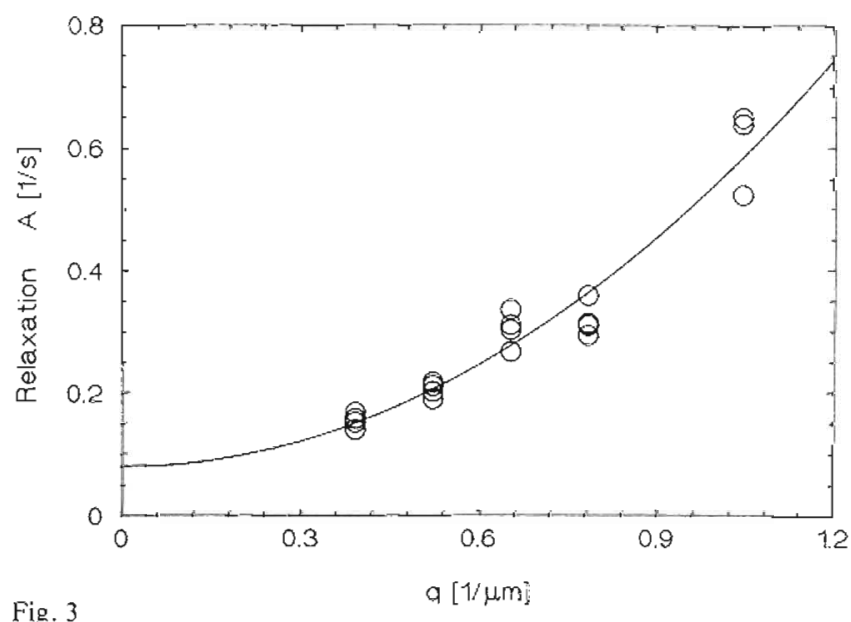

The decay rate $A$ vs. the wave number of the modulation, $q$, for fixed temperature $(170 \mathrm{~K})$. The solid line represents a least squares fit to the data according to $A=a q^{2}+b$ 
$b=\frac{\beta}{\rho} \sqrt{\frac{3}{m} \frac{\mathrm{d} U}{\mathrm{~d} d}} p T^{-3 / 2}$

where $\beta$ is the sticking coefficient of ethanol, $\rho$ the liquid density, $m$ the molecular mass, $d$ the film thickness and $\mathrm{d} U / \mathrm{d} d$ the derivative of the liquid-substrate potential taken at the film surface. In Fig. 4 we plotted the values of $b$ vs. the quantity $p T^{-1.5}$. Satisfactory agreement is found, the straight line representing Eq. 1 . Setting $\beta$ equal 10 one (which seems reasonable at low temperatures) and assuming an inverse power law for the liquid-substrate potential, we obtain a binding energy of aboul $6 \mathrm{meV}$ for ethanol at $30 \AA$ away from the substrate. This is consistent with data reported in the literature [13].

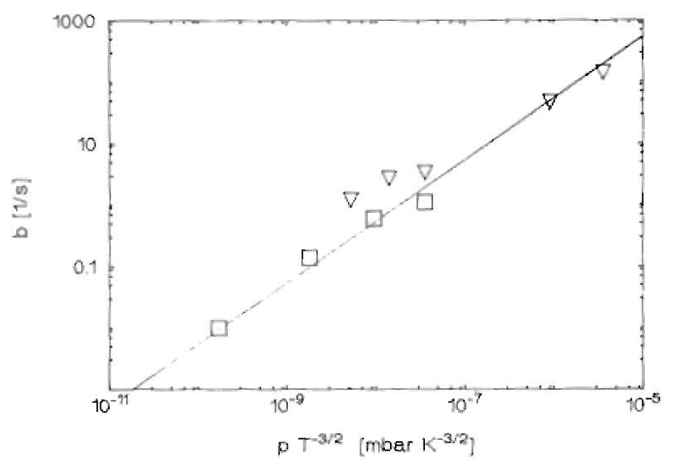

Fig. 4

The coefficient $b$ (decay rate extrapolated to $q=0$ ), obtained for different temperatures. The straight line represents the equilibration rate expected from direct exchange with the vapor phase, as obtained from kinetic gas theory (different symbols represent different experimental runs)

For the coefficient $a$, we adopt the result obtained for Poiseuille flow with no-slip condition [13], which reads

$a=\frac{\rho d^{3}}{3 \eta} \frac{\mathrm{d} U}{\mathrm{~d} d}$

where $\eta$ is the viscosity of the wetting layer. Figure 5 shows the result for the coefficient $a$ in an Arrhenius plot. Two striking features are observed. First, no significant change in the transport behavior occurs in passing the bulk melting point, $T_{\mathrm{m}}$. This indicates that no crystals are lormed and the film remains liquid (ethanol is known to form a glass when cooling is not too slow, with $T_{\mathrm{g}} \approx 90 \mathrm{~K}$ ). Secondly, the lateral transport is almost two orders of magnitude slower than what is expected for viscous flow (solid line, with viscosity data from [15]) in a $30 \hat{\AA}$ thick film.

Several possible explanations for this large discrepancy come to mind. First, since the substrate is formed by an evaporated polycrystalline metal film, the first layers close to the substrate may be pinned by microscopic protrusions owing to the native substrate roughness. In this case, the effective thickness contributing to the flow would be reduced while the van-der-Waals forces remain unchanged, thus

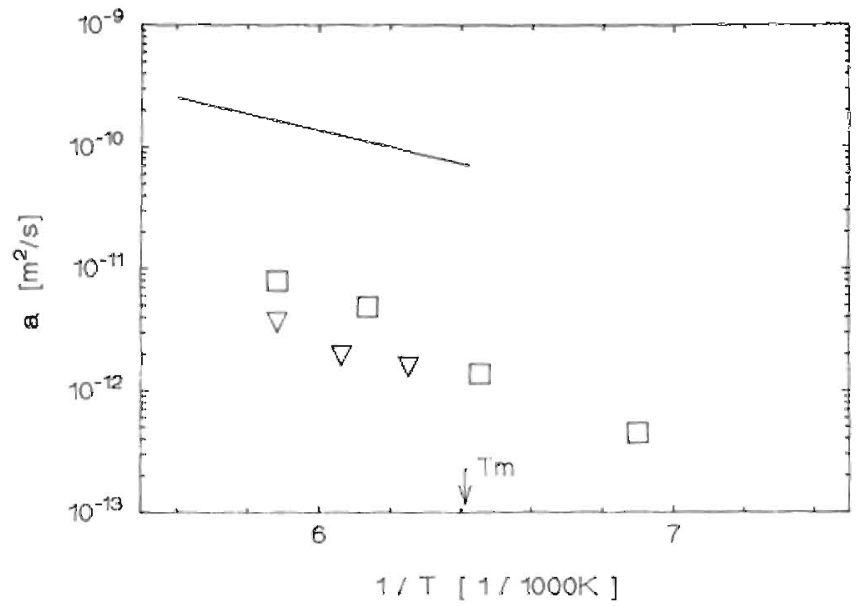

Fig. 5

Arrhenius plot of the coefficient $\alpha$ for two different experimental runs. The solid line corresponds to what is expected for Poiseuille flow, using available data for the viscosity of liquid ethanol. The arrow labeled ' $T_{\mathrm{m}}$ ' indicates the bulk crystallization temperature

strongly reducing $a$. However, scanning tunneling microscopy of our substrates did not reveal a microscopic roughness which could account for this effect. Furthermore, the filsm do not seem to exhibit larger wells necessary for capillary condensation to play a significant role.

Alternatively, the viscosity of the film might be enhanced due to the large van-der-Waals pressure close to the substrate (on the order of $100 \mathrm{bar}$ ), for instance by partial solidification. In fact, it has been observed that the effective viscosity of thin liquid films (between solid walls) may exceed the bulk value by several orders of magnitude [16]. Clearly, this rather spectacular interpretation has to be thoroughly confirmed. Very recent results we obtained with films of liquid propane at somewhat lower temperatures seem to show an enhancement of viscosity comparable to the one found with ethanol.

This work was supported by the Deutsche Forschungsgemeinschaft (SFB 306).

\section{References}

[1] L. Leger, M. Erman, A.M. Guinet-Picard, D. Ausserre, and C. Strazielle, Phys. Rev. Lett. 60, 2390 (1988).

[2] A. M. Cazabat, Adv. Colloid Interface Sci. 34, 73 (1991).

[3] U. Albrecht, A. Otto, and P. Leiderer, Phys. Rev. Lett. 68, 3192 (1992).

[4] G. Reiter, Phys. Rev, Lett, 68, 75 (1992).

[5] J.A. Nieminen, D. B. Abraham, M. Karttunen, and K. Kaski, Phys. Rev. Lett. 69, 124 (1992).

[6] H. Raether, "Surface plasmons on smooth and rough surfaces and on gratings", Springer Tracts in Modern Physics 111 , Springer, Heidelberg, 1988.

[7] J.R. Sambles, G. W. Bradberry, and Fuzi Yang, Contemp. Phys. 32, 173 (1991).

[8] Supplied by Aldrich, unfractionated, with a viscosity of $5 \mathrm{cSt}$. The average molecular weight was $M_{\mathrm{w}}=760$, corresponding to a chain length of 9 monomers.

[9] J.F. Joanny and P.-G. de Gennes, J. Phys. (France) 47, 121 (1986). 
[10] U. Albrecht, PhD Thesis, Konstanz, Germany, 1992.

[11] C. Redon, F. Brochard-Wyart and F. Rondelez, J. Phys. II (France) 2, 1671 (1992).

[12] H. Musil, S. Herminghaus, and P. Leiderer, Surf. Sci. Ler1. 294, L919 (1993).

[13] P.S. Vukusic, G.W. Bradberry, and J.R. Sambles, Surf. Sci. Lett. 277, L34 (1992).

[14] E. Ruckenstein and R. K. Jain, Chem. Soc. Faraday Trans. 270 , $132(1974)$.
[15] "Landoldt-Bornstein; Zahlenwerte und Funktionen", eds. by K. Schäfer and E. Lax, Part II, Vol 2a Berlin 1961.

[16] M.L. Gee, P.M. McGuiggan, J.N. Israelachvili, and A.M. Homola, J. Chem. Plyys. 93, 1895 (1990).

Presented at the Discussion Meeting of the Deutsche Bunsen-Gesellschaft für Physikalische Chemie "Phase Transitions at Interfaces" in Bad Herrenalb, September 22nd to 24 th, 1993 